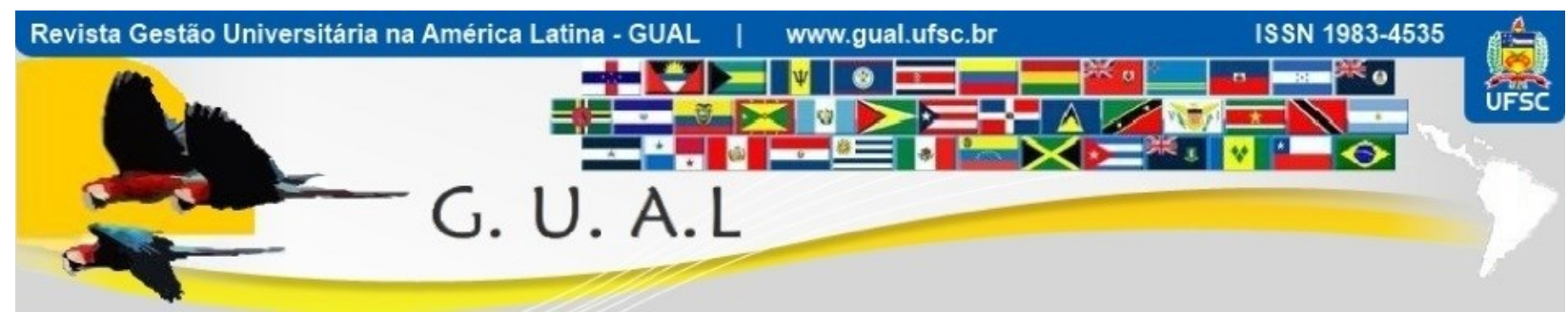

DOI: http://dx.doi.org/10.5007/1983-4535.2019v12n2p160

\title{
REDE DE MOVIMENTAÇÃO DOS SERVIDORES PÚBLICOS CIVIS DA UNIÃO: UM ESTUDO SOB A PERSPECTIVA DA GESTÃO DE PESSOAS NA UFAL
}

\section{CIVIL UNION CIVIL SERVANTS MOVEMENT NETWORK: A STUDY UNDER THE PERSPECTIVE OF PEOPLE MANAGEMENT AT UFAL}

\author{
Polyana Tenório de Freitas e Silva, Mestre \\ https://orcid.org/0000-0002-4492-3865 \\ poly_tenorio@hotmail.com \\ Universidade Federal de Alagoas | Faculdade de Economia, Administração e Contabilidade \\ Maceió | Alagoas | Brasil \\ Milka Alves Correia Barbosa, Doutora \\ https://orcid.org/0000-0002-8114-0333 \\ milka.barbosa@feac.ufal.br \\ Universidade Federal de Alagoas | Faculdade de Economia, Contabilidade e Administração \\ Maceió | Alagoas | Brasil \\ Fernanda Roda de Araujo Cassundé, Doutora \\ https://orcid.org/0000-0002-0621-7505 \\ fernandaroda@gmail.com \\ Universidade Federal do Vale do São Francisco | Colegiado de Administração \\ Petrolina | Pernambuco | Brasil
}

Recebido em 27/julho/2018

Aprovado em 05/fevereiro/2019

Publicado em 02/maio/2019

Sistema de Avaliação: Double Blind Review 


\title{
RESUMO
}

Compete ao Poder Público a organização do seu quadro de servidores, e eventualmente podem ocorrer mudanças que levem a necessidade de remaneja-los. Essa movimentação acontece pelo interesse da administração ou por solicitação do servidor, porém em ambos os casos, a decisão final compete ao próprio ente público que, via de regra, tem discricionariedade para analisar os pedidos de acordo com seus próprios critérios de conveniência e oportunidade. O objetivo deste trabalho foi conhecer como está organizada a rede formada pelos pedidos de remoção realizados pelos servidores técnicos-administrativos da Universidade Federal de Alagoas (UFAL). As temáticas da análise de redes sociais (ARS) e da Gestão de Pessoas (GP) constituíram a base teórica do estudo. A metodologia adotada foi qualitativa e os dados foram coletados por meio de levantamento documental e entrevistas, tendo definido como estratégia o estudo de caso das movimentações dos servidores da UFAL no período de 2012-2016. Os dados demonstram aglomeração de solicitações de remoção para o Campus sede, na capital, sem a devida justificativa, e a permuta entre servidores aparece como uma das principais condições para o deferimento desses pedidos. Como sugestão, propõe um plano de melhoria da rede cujas ações baseiam-se em estratégias de gestão de pessoas.

Palavras-chave: Análise de Redes Sociais. Administração Pública. Gestão de Pessoas. Movimentação de Pessoas.

\begin{abstract}
It's Government's responsibility to organize its staff, and eventual changes may occur, leading to the need of remanue them. This movement is due to the interest of the administration or the request of the server, but in both cases, the final decision lies with the public entity itself, which, as a rule, has discretion to analyze the requests according to their own criteria of convenience and opportunity. The objective of this paper was to know how the network formed by requests for removal carried out by the technical-administrative servers of the Federal University of Alagoas (UFAL) is organized. The topics of social network analysis (SNA) and People Management (GP) were the theoretical basis of the study. The methodology adopted was qualitative and the data were collected through public documents and interviews, having defined as a strategy the study case of the UFAL servers' movements in the period of 2012-2016. The data demonstrates agglomeration of removal requests for the main Campus, in the capital, without proper justification, and the exchange between servers appears as one of the main conditions for granting such requests. As a suggestion, it proposes a network improvement plan whose actions are based on people management strategies.
\end{abstract}

Keywords: Analysis of Social Networks. Public Administration. People Management. Removal. 


\section{REDE DE MOVIMENTAÇÃO DOS SERVIDORES PÚBLICOS CIVIS DA UNIÃO: UM ESTUDO SOB A \\ PERSPECTIVA DA GESTÃO DE PESSOAS NA UFAL \\ DOI: http://dx.doi.org/10.5007/1983-4535.2019v12n2p160}

\section{INTRODUÇÃO}

As pessoas são elemento essencial para o funcionamento de uma organização, isso porque, é a partir delas que as instituições produzem bens e serviços que são oferecidos e geram resultados que impactam diretamente no seu desempenho.

Assim, no âmbito do serviço público, os servidores estão em posição central, pois são eles que levam a efeito os atos do Poder Público, portanto, exige-se a formação e mobilização de competências específicas que mesclam conteúdo técnico e político (BERGUE, 2014).

Apesar disso, a seleção por meio de certame público tem se baseado em cargos e não em competências, o que contribui para se alocar pessoas em áreas com características diversas, sem necessariamente suprir as reais necessidades de competência das atividades típicas do setor onde se está lotado (PANTOJA et al., 2010).

Além da etapa de inserção de novos servidores, as habilidades e características dos recém empossados somente serão avaliadas após um período que permita o reconhecimento de potencialidades, qualificações e outros aspectos relacionados ao comportamento (PIRES et al., 2005). Diante disso, com o passar do tempo, é possível que, das dinâmicas organizacionais ou aspectos individuais do próprio servidor, surja necessidade de promover mudanças com vistas a readequar a força de trabalho e a alinhar a situação particular do servidor público aos objetivos institucionais.

Nesses termos, o regime jurídico único dos servidores públicos civis da União, dispõe sobre as formas de movimentação dos servidores nas organizações públicas, processo que ocorre mediante remoção, redistribuição ou cessão.

Quando se fala em redistribuição e cessão, observar-se uma mudança que envolve mais de uma entidade pública, sendo assim, o servidor é deslocado de uma instituição para outra. A cessão acontece de forma temporária, e a redistribuição, por sua vez, movimenta não apenas o servidor, mas também o próprio código de vaga.

Já a remoção - forma de movimentação de pessoas escolhida como tema central desse trabalho - se trata de uma alteração na lotação do servidor, que permanece no quadro da mesma entidade pública e ocorre de duas formas: a pedido do servidor ou de ofício, quando determinada pela própria administração pública. Em ambos os casos o ato fica condicionado à discricionariedade do Poder Público, que deverá avaliar conveniência e oportunidade. Portanto, a regulamentação de critérios para julgar estes pedidos depende de normas internas 


\section{REDE DE MOVIMENTAÇÃO DOS SERVIDORES PÚBLICOS CIVIS DA UNIÃO: UM ESTUDO SOB A \\ PERSPECTIVA DA GESTÃO DE PESSOAS NA UFAL \\ DOI: http://dx.doi.org/10.5007/1983-4535.2019v12n2p160}

estabelecidas por cada instituição, com exceção dos casos específicos em que o ato administrativo assume caráter vinculatório.

$\mathrm{Na}$ Universidade Federal de Alagoas (UFAL), a organização das demandas de recursos humanos é direcionada à Pró-reitora de Gestão de Pessoas (PROGEP), cuja competência regimental é coordenar políticas de desenvolvimento do corpo docente e técnicoadministrativo, além das atividades relacionadas à administração, ao controle e à avaliação do quadro funcional e dos processos de trabalho. Portanto, a distribuição adequada dos servidores disponíveis na Universidade é atribuição da PROGEP.

A construção de normas para a movimentação de servidores na instituição foi uma ação prevista inicialmente no Plano de Desenvolvimento Institucional (PDI) referente ao quinquênio 2013-2017. Entende-se que o cumprimento da meta proposta no PDI e constante nos relatórios de gestão institucional da UFAL referente à institucionalização de políticas de movimentação dos servidores é necessária à estruturação formal e institucionalização de critérios de remoção da UFAL, pois a ausência dos mesmos quando da apreciação dessas solicitações pode acarretar em pedidos não avaliados adequadamente e em dificuldades de implantar um sistema de gestão por competências, previsto no Decreto 5707/2006 como um dos instrumentos da Política Nacional de Desenvolvimento de Pessoal.

Nesse sentido, ao se verificar a disposição de pessoas e a forma como transitam entre os campi da UFAL, percebeu-se que os processos de remoção sinalizam existência de vínculos de compartilhamento de recursos (humanos) entre as lotações de onde saem e para onde vão os servidores.

Para o estudo desse cenário, o conjunto de indicadores ofertado pela Análise de Redes Sociais (ARS) possibilita compreender aspectos estruturais, relacionais e de centralidade do cenário composto pelo deslocamento de pessoas na instituição, constituída por diversas lotações que, nesse caso, assumem o papel de atores da rede e que, como tal, podem ser estudados pela teoria de redes (FREEMAN, 1979; SCOTT, 2012; BRAND; VERSCHOORE, 2014).

Sendo assim, entende-se que a ARS dispõe de medidas adequadas para diagnosticar padrões e características de uma rede e, com isso, torna-se aplicável ao estudo da movimentação de pessoas na UFAL, considerando que este cenário resulta numa rede passível de análise. 


\section{REDE DE MOVIMENTAÇÃO DOS SERVIDORES PÚBLICOS CIVIS DA UNIÃO: UM ESTUDO SOB A \\ PERSPECTIVA DA GESTÃO DE PESSOAS NA UFAL \\ DOI: http://dx.doi.org/10.5007/1983-4535.2019v12n2p160}

Nessa perspectiva, esta investigação buscou responder ao seguinte questionamento: como está organizado o processamento dos pedidos de remoção que constituem a rede de movimentação de pessoas na UFAL?

A pesquisa traz contribuições teóricas, pois faz uso de conceitos derivados do tema de GP e da ARS, duas áreas bastante relevantes para a Administração Pública que, no entanto, ainda não são abordadas de forma conjunta.

Este artigo foi estruturado em cinco seções, que se iniciam com a presente introdução, seguida da exploração do referencial teórico, na terceira parte é descrita a metodologia adotada, em sequência, são apresentados os resultados e, por último, aparecem as considerações finais.

\section{ANÁLISE DE REDES SOCIAIS (ARS)}

Rede social refere-se a uma tradição nas Ciências Sociais que enfatiza atividades conjuntas e as interações entre participantes em um sistema social (BRAND; VERSCHOORE, 2014).

Do ponto de vista sociológico, as relações estabelecidas podem ser recíprocas ou não, isto significa que as vezes a relação tem um fluxo unidirecional, outras é bidirecional (FIALHO, 2014). Quando a relação tem uma representação gráfica em forma de seta fluindo numa única direção $(\rightarrow$ ou $\leftarrow)$, significa que o laço entre os atores é fraco. Já quando a ligação, que une os nós da rede, tem forma de seta bidirecional $(\leftrightarrow)$, entende-se que o laço é forte, pois é correspondido.

No campo da administração, as redes se formam para integrar organizações, setores e pessoas. Por isso, podem ser classificadas como interorganizacional, intraorganizacional ou intrapessoal, a depender da perspectiva analisada (NOHORIA; ECCLES, 1992). As redes interorganizacionais são compostas por duas ou mais organizações. Já as redes intraorganizacionais, são ligações internas que se formam dentro da mesma organização. Por fim, redes intrapessoais descrevem as relações entre pessoas de determinada organização.

A aplicação do tema não é restrita à esfera privada, visto que, por vezes, o Estado acaba participando de dinâmicas de redes quando atua em parcerias objetivando atingir sua finalidade de forma mais eficaz e abrangente, e em muitos casos, ocupa posição de grande representatividade com papel articulador (OLIVEIRA, 2018). 


\section{REDE DE MOVIMENTAÇÃO DOS SERVIDORES PÚBLICOS CIVIS DA UNIÃO: UM ESTUDO SOB A \\ PERSPECTIVA DA GESTÃO DE PESSOAS NA UFAL \\ DOI: http://dx.doi.org/10.5007/1983-4535.2019v12n2p160}

Vale lembrar que a cooperação é apenas um dos fatores que podem constituir uma rede, pois podem também ser compostas por compartilhamento de informações, recursos e conhecimentos. Em todo caso, os instrumentos que a literatura descreve para estudá-las são indicadores, que medem características específicas.

Estudo realizado por Brand e Verschoore (2014) apresentou as medidas da ARS mais e menos utilizadas pelas pesquisas de redes na área da Administração. O grupo das medidas mais recorrentes refere-se à centralidade de grau, centralidade de intermediação, centralidade de proximidade e densidade; enquanto no grupo das medidas adotadas com frequência intermediária estão: tamanho da rede, clusters, equivalência estrutural e cliques. Por fim, no grupo das medidas pouco ou nunca utilizadas incluem-se poder de Bonacich, transitividade, balanço estrutural, grau dos nós e distância geodésica.

Importa delimitar que, nesta pesquisa, serão adotados os indicadores de tamanho, diâmetro, densidade e centralidade de grau. Os demais indicadores não agregam nem se relacionam com os objetivos propostos neste trabalho.

O tamanho de uma rede é dado pelo número de nodos e indica a probabilidade de interação entre eles (BORDIN et al., 2014). Quanto maior a rede, maior a probabilidade de interação entre os nodos, e menor a possibilidade de um nodo estar conectado com o conjunto total de nodos da rede.

Já o diâmetro, trata-se de medida obtida pelo número de ligações suficientes para ir de um nodo a outro dentro da rede pelo seu caminho mais longo (HANNEMAN; RIDDLE, 2005). Assim, o diâmetro ignora a passagem mais curta, seguindo de um ponto a outro como se as ligações tivessem sempre que passar pelo maior número de atores possível.

A densidade, por sua vez, pode ser calculada dividindo o número de relações existentes entre as possíveis e multiplicando por cem (ALEJANDRO; NORMAN, 2005). Sendo assim, a densidade expõe o potencial de expansão de uma rede.

Por fim, a centralidade de grau, definida por Freeman (1979), compreende duas medidas, podendo indicar tanto o número de ligações estabelecidas por um determinado ator da rede com os demais (grau de saída) como também o número de vínculos cujo fluxo parte de outros atores em direção ao ator central (grau de entrada).

A composição de uma rede surge de situações capazes de agrupar múltiplos atores que compartilham informação, comunicação e recursos. Nesse caso, a movimentação de servidores também compõe uma rede mediante o fluxo de pessoas, as quais representam 


\section{REDE DE MOVIMENTAÇÃO DOS SERVIDORES PÚBLICOS CIVIS DA UNIÃO: UM ESTUDO SOB A \\ PERSPECTIVA DA GESTÃO DE PESSOAS NA UFAL \\ DOI: http://dx.doi.org/10.5007/1983-4535.2019v12n2p160}

recursos humanos, distribuídos e compartilhados entre setores e organizações públicas. Sendo assim, para o desenvolvimento da atual pesquisa, além da análise de redes, foi necessário perceber algumas questões quanto ao gerenciamento de pessoas e suas peculiaridades na Administração Pública.

\section{GESTÃO DE PESSOAS (GP) E MOVIMENTAÇÃO DE SERVIDORES PÚBLICOS}

As pessoas constituem elemento central das organizações, pois os seus resultados são diretamente dependentes das equipes que desenvolvem e executam atribuições prédeterminadas (BERGUE, 2014). Assim, escolher as pessoas adequadas para cada função representa vantagem estratégica para a organização.

No setor público, a gestão de pessoas assume certa complexidade na medida em que lida com contingenciamento de despesas, necessidade de demonstrar o interesse público em cada ato, impossibilidade de atuar fora dos padrões burocráticos previstos pela legislação, entre outros (AMARAL, 2006; ABREU; GUIMARÃES, 2014). A Administração Pública brasileira ainda é dominada pela rigidez formal, haja vista que os cargos são definidos com atribuições muito especificas, e o servidor que ingressa para desempenhar determinada função não pode ser deslocado para outra, sob pena de os administradores cometerem uma inconstitucionalidade (PANTOJA et. al, 2010).

De fato, alguns aspectos dificultam o recrutamento do capital humano qualificado e o gerenciamento de recursos humanos nas organizações públicas: a visão de que o servidor é um custo substancial e não um recurso a ser estrategicamente utilizado, a estrutura burocrática e a rigidez das regas, contingenciamento de recursos, limitação do uso do sistema de recompensas e, falta de liderança adequada, pois a indicação de pessoas para ocupar tal posição é, via de regra, uma decisão de natureza política (INGRAHAM; RUBAII, 2007).

Essas restrições atrapalham a implementação de um modelo de gestão de pessoas baseado em competências, porquanto qualquer estratégia adotada encontra barreiras nas questões retro mencionadas, e por isso, não raro se desenvolvam mecanismos que possibilitem o deslocamento intersetorial de servidores no âmbito das organizações públicas, como forma de atenuar as limitações burocráticas que atrapalham a estruturação do quadro de pessoal no setor público. Por essa razão, a movimentação de pessoas é considerada como um dos três processos que compõem a gestão de pessoas (DUTRA, 2013). 


\section{REDE DE MOVIMENTAÇÃO DOS SERVIDORES PÚBLICOS CIVIS DA UNIÃO: UM ESTUDO SOB A \\ PERSPECTIVA DA GESTÃO DE PESSOAS NA UFAL \\ DOI: http://dx.doi.org/10.5007/1983-4535.2019v12n2p160}

Os institutos que permitem a movimentação dos servidores públicos são tratados pela Lei 8112/90, que regulamenta o regime jurídico dos servidores públicos civis da União. A mobilidade dos servidores encontra respaldo nos artigos 36 e 37 da já mencionada lei, que remetem, respectivamente, à remoção e à redistribuição.

A remoção é definida na legislação como o deslocamento do servidor, a pedido ou de ofício, no âmbito do mesmo quadro, com ou sem mudança de sede (BRASIL, 1990). Ou seja, o ato tanto pode partir de uma solicitação feita pelo servidor quanto de uma deliberação da própria gestão. No entanto, mesmo quando se fala em remoção a pedido, ainda sim entende-se que a Administração Pública exerce, geralmente, discricionariedade no momento do deferimento ou indeferimento do pedido.

Como a remoção ocorre dentro do mesmo quadro, o servidor passa a exercer suas atividades em outra unidade do mesmo ente público ao qual pertence. Nesse caso, pode ou não resultar numa mudança de domicílio do servidor, uma vez que, a lotação de destino pode estar situada na mesma cidade ou sediada em outro município.

Existem outros aspectos da administração pública que podem ser estudados pela ARS e pela GP, mas que, no entanto, não foram abordados nesse referencial teórico devido ao escopo da pesquisa limitar-se à rede construída movimentação de servidores que transitam entre suas lotações exclusivamente por meio da remoção. Uma vez delimitado o objeto de estudo, no capítulo seguinte, será demonstrado o procedimento adotado para coleta, descrição e análise dos dados.

\section{PERCURSO METODOLÓGICO}

A presente investigação adotou abordagem qualitativa, cuja escolha foi motivada principalmente pelo entendimento de que as pesquisas qualitativas se preocupam com o aprofundamento da compreensão de um grupo de indivíduos, seja esse um grupo social, ou uma organização (GERHARDT; SILVEIRA, 2009). Nesse sentido, estudos que tendem a acompanhar o significado que as experiencias e interações tem para as pessoas em seu próprio ambiente social classificam-se como pesquisas qualitativas interpretativas (MERRIAM, 2002).

A pesquisa procurou analisar, com base em indicadores, as características da rede de movimentação de pessoas na UFAL. Adotar uma abordagem qualitativa, trouxe aprofundamento na compreensão de alguns fenômenos observados no levantamento de 


\section{REDE DE MOVIMENTAÇÃO DOS SERVIDORES PÚBLICOS CIVIS DA UNIÃO: UM ESTUDO SOB A \\ PERSPECTIVA DA GESTÃO DE PESSOAS NA UFAL \\ DOI: http://dx.doi.org/10.5007/1983-4535.2019v12n2p160}

dados: inexistência de processos de remoção de ofício, migração de técnicos em busca de cursos para progredir na carreira, ausência de critérios para apreciação dos pedidos de remoção e para definir lotação inicial.

A pesquisa incluiu o caso de remoção dos servidores técnicos-administrativos da UFAL compreendidos no período entre 2012 e 2016, totalizando 135 pedidos. Este recorte de tempo foi definido em razão da disponibilidade de dados junto à PROGEP, setor responsável pela gestão de pessoas na universidade, pois este informou que levantamentos anteriores ao período estabelecido poderiam resultar em dados fragmentados devido ao prazo máximo de guarda de documentos desta natureza.

Para a pesquisa, foram considerados os processos onde o servidor solicita remoção para outra cidade excluindo-se remoções dentro do mesmo município, isso porque, processos de remoções intermunicipais têm implicações tanto para o servidor, como para o próprio Poder Público, que assume obrigação de pagar ajuda de custo para compensar despesas de instalação desse servidor e de sua família na localidade da nova lotação quando o deslocamento ocorre com mudança de domicílio por interesse da Administração.

Importante esclarecer que não se incluiu casos de movimentação de docentes pois para tal cargo há uma área de atuação determinada em concurso, o que restringe a possível lotação ou o processo de remoção.

Adotou-se três métodos de coleta de dados, nomeadamente, análise documental, entrevistas informais e semi-estruturadas. A escolha dessas técnicas de coleta levou em conta o tipo de estudo que se desejava realizar, a natureza do problema, o nível de aprofundamento desejado e o cuidado metodológico necessário para conferir credibilidade, confiabilidade e autenticidade à pesquisa.

Para Collis e Hussey (2005), os dados secundários são dados que já existem, como livros, documentos (por exemplo, estatísticas publicadas, relatórios anuais e contas de empresas, e registros internos mantidos por organizações, como registros de pessoal) e filmes. Com essa orientação, foram analisados os relatórios de gestão da UFAL dos anos de 2012 a 2016, o plano de desenvolvimento institucional vigente para o período de 2013 a 2017, além dos dados estatísticos sobre os pedidos de remoção da instituição.

Já a entrevista informal, definida como a menos estruturada possível e recomendada para abordar realidades pouco conhecidas ou para oferecer visão aproximativa do problema (BRITTO JÚNIOR; FERES JÚNIOR, 2011), procedeu-se mediante visita in loco à PROGEP, 


\section{REDE DE MOVIMENTAÇÃO DOS SERVIDORES PÚBLICOS CIVIS DA UNIÃO: UM ESTUDO SOB A \\ PERSPECTIVA DA GESTÃO DE PESSOAS NA UFAL \\ DOI: http://dx.doi.org/10.5007/1983-4535.2019v12n2p160}

objetivando verificar a existência de regulamento, resolução ou portaria expedida pela UFAL a respeito de critérios formais para apreciação dos pedidos de remoção dos servidores técnicos na instituição.

Como última etapa, foram realizadas entrevistas semiestruturadas, definindo-se no roteiro os temas a serem explorados, e eventuais perguntas, mantendo-se a flexibilidade no modo como as perguntas são formuladas e a ordem pela qual aparecerem (FONSECA, 2002). Quanto a seleção de servidores entrevistados, foram escolhidos dois respondentes lotados na PROGEP, aqui denominados E1 e E2, que atuam respectivamente com o recrutamento e a movimentação de pessoas, duas funções que se alinham com aspectos observadas pela pesquisa, portanto, trata-se de uma escolha intencional (OLIVEIRA, 2011).

Após a coleta de dados, passou-se a etapa de análise. Inicialmente, foi necessário compor uma matriz que pudesse ser processada para delinear a rede de movimentação de servidores. Para isso, o fator chave recaiu sobre a reciprocidade entre os processos de remoção de um polo da UFAL para outro, de forma que os atores da rede ficaram definidos pelas cidades onde estão situados os polos.

A base dessa matriz foram os dados de remoção na UFAL, cedidos pela gestão. Os registros institucionais foram disponibilizados em planilha formatada no software Microsoft Excel, com informações sobre pedidos recebidos, atendidos, negados e pendentes, bem como, as razões pelas quais os servidores pedem remoção.

Os dados foram processados no software UCINET for Windows, cuja escolha foi influenciada pelas opções referentes aos níveis de análise de redes constantes no programa, que contempla todos os indicadores selecionados para este estudo específico: tamanho, diâmetro, densidade, centralidade de grau (HANNEMAN; FREEMAN, 1979; ALEJANDRO; NORMAN, 2005; RIDDLE, 2005; BORDIN et al., 2014).

A matriz construída para formar uma rede no UCINET foi binária (ALEJANDRO; NORMAN, 2005), por isso, dentro dela, as intersecções entre os atores podem receber apenas dois valores distintos: 0 ou 1. Sendo assim, caso existisse solicitação de remoção de uma cidade para a outra, valor atribuído seria 1 , de outra forma, se não existissem pedidos entre essas cidades, o valor atribuído seria 0 .

Todas as cidades onde há campi da UFAL se cruzam duas vezes na matriz. Se nessas duas vezes em que elas se encontram o valor for 1 , significa que o vínculo é recíproco (ligação forte). Se nas intersecções aparecer 0 em uma e 1 na outra, significa que o laço é 


\section{REDE DE MOVIMENTAÇÃO DOS SERVIDORES PÚBLICOS CIVIS DA UNIÃO: UM ESTUDO SOB A \\ PERSPECTIVA DA GESTÃO DE PESSOAS NA UFAL \\ DOI: http://dx.doi.org/10.5007/1983-4535.2019v12n2p160}

unidirecional (ligação fraca). Se o valor for 0 para as duas, quer dizer que as cidades não estão conectadas entre si (ligação inexistente).

Importante ressaltar que, a PROGEP também foi inserida na rede, tendo em vista que todos os processos de remoção precisam, obrigatoriamente, passam por ela, pois este setor é o responsável por administrar, controlar e avaliar o quadro funcional e os processos de trabalho. Dessa forma, a PROGEP mantém vínculos com todos os demais atores da rede, e por essa razão, atribuiu-se o valor 1 em todas as suas intersecções.

\section{DISCUSSÃO DOS DADOS}

A instituição investigada conta com mais de 3.302 servidores ativos e divide-se em três campi. O campus principal, A.C. Simões, localiza-se em Maceió, e abrange centros, institutos, pró-reitorias e órgãos de apoio na capital. Já o campus Arapiraca, o segundo maior da UFAL, subdivide-se em quatro polos situados nos municípios de Arapiraca, Viçosa, Penedo e Palmeira dos Índios. Por fim, o campus do Sertão inclui os polos de Delmiro Gouveia e Santana do Ipanema. Dessa forma, a instituição faz-se presente em significante parte da extensão territorial do estado de Alagoas, uma vez que além da capital, atua também em regiões na fronteira do estado, como Penedo (170km de Maceió) e Delmiro Gouveia (300km de Maceió).

Para atingir o objetivo proposto pela pesquisa, a análise dos dados coletados foi esquematizada na seguinte ordem: aspectos gerais da rede, descrição da rede, distância social média entre atores, potencial de crescimento da rede, importância da PROGEP enquanto ator e comparação entre polos ou unidades de ensino da UFAL.

\subsection{ASPECTOS GERAIS DA REDE}

Os dados apontam para uma predominância de solicitações de remoção para o campus sede, em Maceió. Na situação oposta, encontra-se Arapiraca, cidade que com o maior número de processos de remoção protocolados. Apesar disso, Arapiraca ainda é um dos grandes centros da UFAL, pois mesmo que seja responsável por 32 solicitações de remoção protocoladas, também é o polo com o segundo maior número de pedidos recebidos, ficando atrás apenas de Maceió nesse quesito. Vale salientar, também, que 31 (dentre 32) dos pedidos de remoção provenientes de Arapiraca solicitam transferência para campus sede, em Maceió, enquanto que todos os demais polos têm processos de remoção abertos tanto para Maceió 


\section{REDE DE MOVIMENTAÇÃO DOS SERVIDORES PÚBLICOS CIVIS DA UNIÃO: UM ESTUDO SOB A \\ PERSPECTIVA DA GESTÃO DE PESSOAS NA UFAL \\ DOI: http://dx.doi.org/10.5007/1983-4535.2019v12n2p160}

quanto para Arapiraca. Nesse sentido, é factível admitir a existência de um fluxo migratório para os servidores, pois os pedidos de remoção seguem um padrão de deslocamento dos municípios mais afastados da capital em direção aos mais próximos, até chegar ao campus A.C. Simões.

Também é possível observar que praticamente não existem pedidos de servidores almejando remoção para Delmiro Gouveia e Santana do Ipanema. Essa situação torna improvável a ocorrência de eventuais permutas dificulta que os processos de remoção originados nas cidades mencionadas se consolidem. Para minimizar esse efeito negativo, é preciso tornar as unidades do interior atrativas aos servidores, promovendo incentivo à permanência nos municípios e ampliando o interesse de remoções para esses locais.

Quando questionados acerca das medidas da gestão para fixar os servidores nos polos do interior, os entrevistados E1 e E2 apontaram que a UFAL tem adotado a prática de estipular em edital, um prazo mínimo de permanência na lotação inicial. De acordo com E2 "isso não impede que eles venham a protocolar os pedidos, mas a gestão tem poder para indeferir o pleito com base nas regras previstas pelo próprio edital". Contudo, verificou-se que, apesar dessa medida, o número de processos pendentes (33) se apresenta superior aos pedidos indeferidos (2), portanto, mesmo dispondo dessa fundamentação, a gestão não vem negando pedidos de remoção, apenas acumulando-os.

Os dados coletados sinalizam que a movimentação de pessoas na UFAL ainda está longe de se adequar às práticas definidas por Dutra (2013). Alguns dos problemas encontrados foram na captação, que esbarra em limites legais; na internalização dos servidores, que não promove o alinhamento da atuação com os conhecimentos individuais de cada um; e nas transferências, que não obedecem a critérios objetivos.

\subsection{DESCREVENDO A REDE}

As informações sobre as lotações de origem e de destino de cada pedido de remoção protocolado permitiram montar uma matriz para compor o desenho da rede de movimentação de servidores da UFAL. Os polos e unidades de ensino de cada município em que a instituição se faz presente são os atores dessa rede. A inclusão de um vértice próprio para a PROGEP na rede construída, explica-se pelo fato dela controlar todas as ações de movimentação de pessoas dentro da universidade. Os vínculos da rede foram tratados com 


\section{REDE DE MOVIMENTAÇÃO DOS SERVIDORES PÚBLICOS CIVIS DA UNIÃO: UM ESTUDO SOB A \\ PERSPECTIVA DA GESTÃO DE PESSOAS NA UFAL \\ DOI: http://dx.doi.org/10.5007/1983-4535.2019v12n2p160}

valores 0 e 1, baseado nos pedidos de remoção protocolados entre atores, portanto, o preenchimento binário da matriz segue o manual de Alejadro e Norman (2005).

As informações foram processadas e desenhadas por softwares específicos, respectivamente UCINET e NetDrawer, o que permitiu construir uma reprodução gráfica e extrair indicadores da rede estudada, conforme ilustra figura 01.

Figura 01 Rede de movimentação de servidores da UFAL

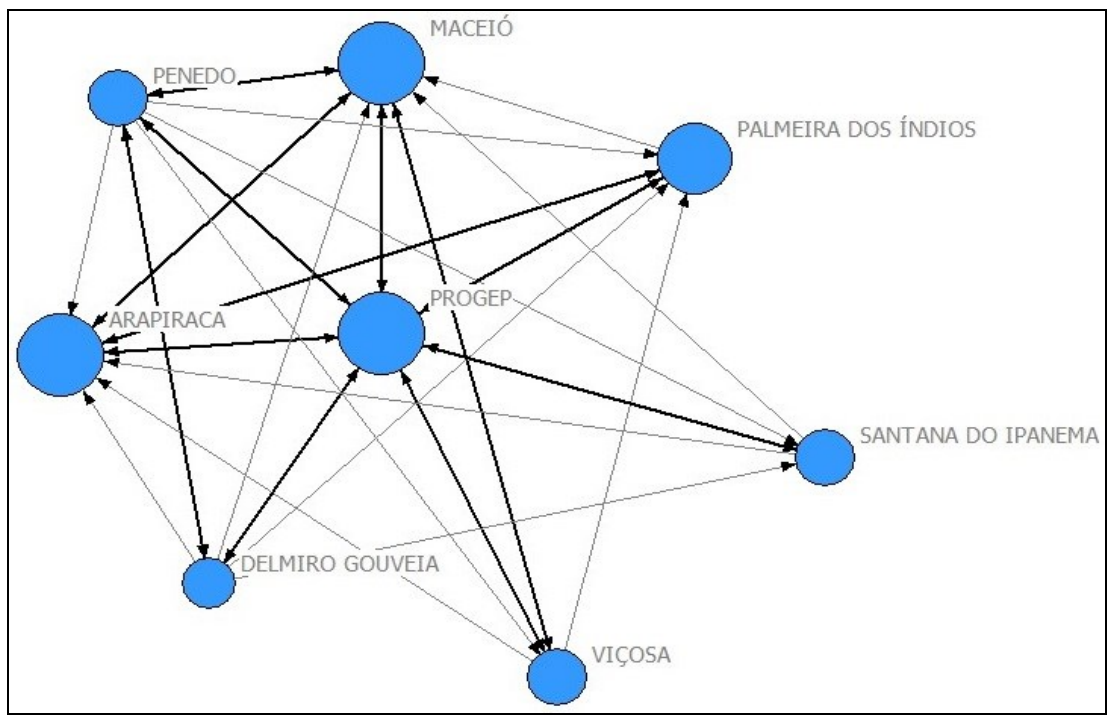

Fonte: Dados da pesquisa.

É possível observar diferentes tamanhos entre os atores. Isso ocorre devido a diferença existente entre o número de setas que transitam entre os vértices, sendo assim, o tamanho do ator está diretamente ligado ao interesse dos servidores de outros polos em ser removidos para lá. Quanto menor o ator, menos pedidos de remoção de outros polos ele recebe. Uma propriedade do UCINET capaz de definir o tamanho de cada vértice de acordo com os seus respectivos graus de entrada (FREEMAN, 1979).

A rede destaca também alguns vínculos mais escuros e espessos do que os outros. Estes representam os laços bidirecionais, isto é, ligações entre polos cujos pedidos de remoção não apenas são recebidos, mas também solicitados entre si. Dessa forma, os atores ligados por traços mais escuros têm uma probabilidade maior de sucesso nas remoções solicitadas entre eles, pois a reciprocidade do vínculo torna mais propícia a permuta de servidores, que é um fator que facilita o deferimento dos pedidos, sendo tal premissa confirmada pelo entrevistado E1, ao afirmar que remoções sem permuta requerem analise sobre prejuízo ao princípio da continuidade do serviço público. 


\section{REDE DE MOVIMENTAÇÃO DOS SERVIDORES PÚBLICOS CIVIS DA UNIÃO: UM ESTUDO SOB A \\ PERSPECTIVA DA GESTÃO DE PESSOAS NA UFAL \\ DOI: http://dx.doi.org/10.5007/1983-4535.2019v12n2p160}

Os gestores têm dado preferência pelas remoções com permuta de servidores ao invés de receber um código de vaga em troca do técnico removido. Porém, a rede possui apenas 12 vínculos bidirecionais, o que significa que a maior parte das remoções protocoladas não propõe troca de pessoal. A falta de reciprocidade reflete numa menor densidade na rede (BRAND; VERSCHOORE, 2014), conforme será descrito adiante.

Diante da conjuntura descrita, a permuta entre servidores parece ser o método de remoção mais eficiente para todos os envolvidos, pois a reposição imediata de pessoal permite que a gestão mantenha o dimensionamento institucional, que os setores não fiquem com o funcionamento comprometido, e que os solicitantes tenham seus pedidos de movimentação atendidos em um prazo menor.

\subsection{DISTÂNCIA SOCIAL MÉDIA ENTRE ATORES}

O caso em análise resulta em uma rede de oito atores, valor menor do que seria obtido se o estudo descesse a nível de rede intrapessoal, onde a rede seria composta por 135 atores, pois cada servidor solicitante de remoção passaria a ter o status de nó. Portanto, a rede intraorganizacional da movimentação de servidores na UFAL tem tamanho relativamente pequeno, se comparada a rede intrapessoal. Nesse sentido, quando se trabalha com uma rede pequena, ela pode ser mais facilmente gerenciada, visto que existem poucos atores para coordenar. Outra vantagem de uma rede pequena é o aumento da possibilidade de conexão entre todos os nodos devido as poucas opções de vínculos (BORDIN et. al., 2014).

A partir desse entendimento, nota-se que observar ou provocar interações entre os atores da rede intraorganizacional formada pelos polos e unidades de ensino da UFAL é mais fácil do que a rede intrapessoal constituída pelos servidores interessados na remoção. Dessa forma, a rede ser melhor administrada pela PROGEP caso esta mantenha o foco das ações mais direcionadas para os polos e unidades do que sobre os servidores. Além disso, redes pequenas facilitam a formação de ligação entre atores, que no caso em estudo, pode fazer toda a diferença em termos de remoção, pois vínculos bidirecionais/recíprocos entre todos os polos da universidade ampliam a probabilidade de reposição do servidor removido por outro.

Com relação ao diâmetro da rede, o UCINET chegou ao valor 2, sendo este o número da distância social máxima entre os atores mais afastados. Uma boa parte dos atores da rede, estão conectados entre si por um vínculo direto e, aqueles que não estão, mantém uma relação 


\section{REDE DE MOVIMENTAÇÃO DOS SERVIDORES PÚBLICOS CIVIS DA UNIÃO: UM ESTUDO SOB A \\ PERSPECTIVA DA GESTÃO DE PESSOAS NA UFAL \\ DOI: http://dx.doi.org/10.5007/1983-4535.2019v12n2p160}

intermediada pela PROGEP, pois esta constitui uma ponte entre os demais atores devido ao seu papel nos processos de remoção.

Segundo o entrevistado E1, "existem duas coordenadorias situadas em Arapiraca e Delmiro (Gouveia) que funcionam como uma extensão da PROGEP, elas facilitam o trabalho com os servidores fora do A.C. Simões". Esse é um fator que aproxima ainda mais as lotações do interior com a pró-reitoria, e consequentemente não permite que os demais vértices se dispersem, reforçando o entendimento de que o resultado de diâmetro 2 pode ser atribuído a sua atuação na rede.

Seria ideal que a distância máxima entre atores fosse 1, assim todos estariam diretamente ligados, e esse tipo de interação poderia promover e dar celeridade ao andamento dos processos de remoção, uma vez que tornaria mais fácil a possibilidade de encontrar alguém para proceder com a remoção por meio de permuta, o que evitaria que os processos ficassem pendentes. Entretanto, o fato da rede ter um diâmetro de 2 ligações significa que nos casos onde não existe conexão de grau 1, a PROGEP desempenha o importante papel de intermediar os vínculos para que não passem de 2 graus de distância.

\subsection{POTENCIAL DE CRESCIMENTO DA REDE}

A nível relacional, analisou-se a densidade da rede. No caso estudado, o valor da densidade encontrado para a rede analisada foi de 0,661 , número que indica potencial de crescimento da rede, seja em função da formação de novos vínculos, seja pela transformação de ligações unidirecionais em recíprocas. Quanto mais o resultado se aproxima de 1, mais densa é a rede e, consequentemente, menor é o seu potencial de expansão, pois os atores já têm muita interação (BRAND; VERSCHOORE, 2014).

É importante observar, contudo, que outras medidas além dessa ampliação precisam ser adotadas, tais como melhorar a estrutura e as condições de trabalho dos polos, ofertar opções para qualificação dos técnicos no interior, fazer levantamento do perfil dos servidores e direcioná-los para atribuições adequadas às suas habilidades, pois estes são fatores que, assim como a possibilidade de permuta, também exercem influência sobre as dinâmicas da rede.

Além disso, o deferimento dos pedidos, via de regra, precede de reposição de pessoal ou, pelo menos, da manutenção do cargo vago até que ocorra provimento da vaga. Corroborando, a própria gestão relata dificuldades em movimentar pessoas quando não ocorre 


\section{REDE DE MOVIMENTAÇÃO DOS SERVIDORES PÚBLICOS CIVIS DA UNIÃO: UM ESTUDO SOB A \\ PERSPECTIVA DA GESTÃO DE PESSOAS NA UFAL \\ DOI: http://dx.doi.org/10.5007/1983-4535.2019v12n2p160}

a substituição imediata do servidor removido, pois as nomeações para códigos de vaga vazios dependem da existência de cadastros de reserva de concursos vigentes, instrumento que se tornou escasso devido ao Anexo II do Decreto 6.944/2009.

Assim, verifica-se que as remoções constituem parte do sistema de gestão de pessoas na administração pública e, portanto, são atos complexos que prescindem de aspectos como análise de perfis para adequar a universidade a um modelo de gestão por competências, levantamento dos motivos que justifiquem a medida, e manutenção do dimensionamento do quadro da instituição.

Nesse sentido, é justificável inserir a permuta entre servidores como um dos critérios para avaliar os pedidos de remoção. Contudo, para que se possa exigir isso, a gestão precisa proporciona meios para que se tenha conhecimento sobre outros servidores interessados em permutar, uma ação que ainda não foi devidamente estruturada na UFAL. Sobre esse aspecto, o entrevistado E1 relatou que a consulta direta à PROGEP é o meio pelo qual os servidores tomam ciência das permutas disponíveis na instituição: "entram como possíveis permutas o estoque de pedidos que não foram atendidos, a partir disso a PROGEP identifica as oportunidades (de permuta) e intermedia a remoção" (E1). O entrevistado E2, por sua vez, explicou que a gestão pensa em "montar um banco de permutas para facilitar essa visualização, porque as vezes, um servidor tem interesse na remoção e não sabe que outro também tem, isso dificulta bastante o nosso trabalho".

Contudo, é preciso que essa ideia deixe de ser uma mera intenção, e passe a ser de fato executada, pois é uma medida que pode ser classificada como parte de uma política de movimentação de servidores na UFAL, a qual necessita de implementação iminente devido a previsão de metas do PDI 2013-2017, cujo descumprimento pesa de forma negativa em eventual auditoria realizada pelo órgão de controle estatal responsável por fiscalizar a instituição.

O fato da rede ainda não ter atingido seu potencial pleno, limita a interação entre polos/unidades e como consequência, os servidores têm pouca ou nenhuma informação sobre pessoas em outras lotações, que é um pressuposto básico para celebrar permutas. No caso da movimentação de pessoas da UFAL, existem atores que não tem vínculo direto entre si, como acontece entre Delmiro Gouveia e Viçosa, ou com Santana do Ipanema e Palmeira dos Índios, ou mesmo com Viçosa e Santana do Ipanema. 


\section{REDE DE MOVIMENTAÇÃO DOS SERVIDORES PÚBLICOS CIVIS DA UNIÃO: UM ESTUDO SOB A \\ PERSPECTIVA DA GESTÃO DE PESSOAS NA UFAL \\ DOI: http://dx.doi.org/10.5007/1983-4535.2019v12n2p160}

A partir do momento em que os servidores tomarem conhecimento da possibilidade de remoção com permuta entre as lotações, os vínculos podem se tornar recíprocos de forma espontânea, porém isso demandará uma ação por parte da gestão no sentido de divulgar e não apenas manter registros dos pedidos de remoção desses locais. A densidade da rede também é afetada pela reciprocidade das ligações, portanto, além de construir novos vínculos, é preciso fortalecer aqueles já existentes, para que se tornem bidirecionais, algo que deve acontecer junto com o surgimento das novas conexões.

\subsection{IMPORTÂNCIA DA PROGEP ENQUANTO ATOR NA REDE}

Em relação a centralidade, definida por Freeman (1979) como posição marcada pela menor distância possível de todos os outros pontos, buscou-se medir o grau de entrada e de saída dos atores na rede de movimentação dos servidores da UFAL. Quanto maior o grau de entrada, maior é a procura de uma determinada localidade como destino das remoções. Em contraponto, um alto grau de saída significa grande concentração de pedidos de remoção solicitando sair daquela lotação. Na tabela 01 é possível observar os valores de centralidade encontrados para cada município.

Tabela 01 Centralidade de Grau

\begin{tabular}{lccc}
\hline \multirow{2}{*}{ Atores } & \multicolumn{3}{c}{ Centralidade de Grau } \\
\cline { 2 - 4 } & Grau de entrada & Grau de saída & Total \\
\hline PROGEP & 7 & 7 & 14 \\
Maceió & 7 & 4 & 11 \\
Arapiraca & 7 & 3 & 10 \\
Viçosa & 3 & 4 & 7 \\
Penedo & 3 & 7 & 10 \\
Palmeira dos Índios & 5 & 3 & 8 \\
Delmiro Gouveia & 2 & 6 & 8 \\
Santana do Ipanema & 3 & 3 & 6 \\
\hline
\end{tabular}

Fonte: Dados da pesquisa.

Especificamente se tratando da PROGEP, a centralidade de grau assume outra interpretação, pois os graus de entrada e saída não indicam pedidos de remoção originados e recebidos, mas sim os processos dessa natureza cuja tramitação se deu por meio deste ator. Além disso, verifica-se que o grau de entrada da PROGEP na rede é igual àquele visualizado em Maceió e Arapiraca, o que, por si só já a torna um ator bastante central na rede, porém essa centralidade fica ainda mais evidente quando se fala em grau de saída. Isso porque, a próreitoria mantém reciprocidade em todos os seus vínculos, portanto, é o único até com valores iguais de entrada e de saída. 


\section{REDE DE MOVIMENTAÇÃO DOS SERVIDORES PÚBLICOS CIVIS DA UNIÃO: UM ESTUDO SOB A \\ PERSPECTIVA DA GESTÃO DE PESSOAS NA UFAL \\ DOI: http://dx.doi.org/10.5007/1983-4535.2019v12n2p160}

Isso confirma o resultado esperado para a centralidade da PROGEP, situação que corrobora as atividades institucionais a ela atribuídas, as quais possibilitam a este ator uma tomada de decisões mais consistentes sobre processos de trabalho, pois interage com as realidades de todos os demais atores com quem mantém vínculos, o que permite buscar alternativas de alocação de servidores por competências, ao tempo em que coordena ações orientadas pelas políticas institucionais, necessidades dos servidores e limites da legislação.

Verificou-se, ainda, que teoricamente, ao retirar a PROGEP da rede, não haveria prejuízo a manutenção do fluxo de remoções pois trata-se de uma rede distribuída. Contudo, isso poderia resultar em pedidos de remoção que ficariam descoordenados e o controle do quadro funcional da instituição caótico, posto não haveria dimensionamento adequado da força de trabalho, já que nem as lotações nem os próprios servidores tem uma noção geral sobre as necessidades de capital humano da universidade como um todo.

Portanto, na perspectiva da teoria de redes, a movimentação de pessoas na UFAL pode se manter funcional com a eliminação da PROGEP devido a distribuição não centralizada dos vínculos, como descreve o estudo de Baran (1964). Entretanto, no aspecto da gestão de pessoas, entende-se que esta é uma área que precisa existir de forma integrada, e o conjunto de políticas e práticas que a formam deve, a um só tempo, atender aos interesses e expectativas da organização e das pessoas (DUTRA, 2013). Dessa forma o gerenciamento de RH não é passível de ser desenvolvido individualmente pelos setores da UFAL, pois perderia o elemento da integração, algo que a PROGEP, na condição de mediador das relações intersetoriais pode garantir.

\subsection{COMPARAÇÃO ENTRE POLOS/UNIDADES DE ENSINO}

Por não dispor de critérios objetivos definidos oficialmente para análise dos pedidos de remoção, verificou-se que a UFAL adotou a prática de processar pedidos associados a uma permuta entre servidores das lotações de origem e de destino, pois assim pode manter o quadro de pessoal dentro do dimensionamento já existente. Quanto aos demais processos, que não apresentam proposta de troca de servidores, ficam pendentes até que o requisito da permuta seja atendido ou até ocorrer desistência do pedido.

Dos processos analisados, 67 não expressavam o que motivou e remoção. Essa é uma quantidade expressiva dentro da amostra analisada, levando em consideração que corresponde praticamente à metade do levantamento total. Na visão do entrevistado E2, esse problema da 


\section{REDE DE MOVIMENTAÇÃO DOS SERVIDORES PÚBLICOS CIVIS DA UNIÃO: UM ESTUDO SOB A \\ PERSPECTIVA DA GESTÃO DE PESSOAS NA UFAL \\ DOI: http://dx.doi.org/10.5007/1983-4535.2019v12n2p160}

falta de exposição de motivos para remoções, assim como a ausência de definição de lotação de destino podem ocorrer devido à falta de um formulário específico para solicitar remoção.

As 68 remoções que apresentavam justificativas foram categorizados em quatro grupos: a) motivos de saúde; b) incompatibilidade das atribuições com a formação do servidor; c) estudos e qualificação; d) questões de ordem pessoal (família, residência, ameaça, etc.). Dentre as justificativas apresentadas, há apenas duas situações em que a Universidade pode efetivamente interferir, quais sejam, remoção para estudos e qualificação e atribuições incompatíveis com a área de formação do técnico.

Em relação às oportunidades de qualificação entre os municípios abrangidos pela UFAL, nota-se uma predominância de ofertas de cursos no campus A.C. Simões. Essa constitui uma das justificativas para a alta demanda de pedidos de remoção do interior para a capital, pois $9 \%$ dos processos apresentam motivo relacionado a estudos e qualificação.

Pensar em formas para que os servidores se qualifiquem sem precisar sair de suas lotações pode reduzir a demanda por remoções, na medida em que concede a oportunidade dos técnicos atingirem novos padrões na carreira em sua localidade. Dando suporte a essa constatação, o entrevistado E2 afirmou que: "as pessoas procuram o A.C. Simões pelo que o campus proporciona, é claro que muitas remoções estão ligadas a questões pessoais do servidor, mas também é alta a demanda por qualificação".

Além disso, considerando que o acesso à rede de ensino é um dos fatores que incidem sobre o cálculo do IDH, admite-se que a discussão dessas questões vai além de uma proposta meramente organizacional para capacitar técnicos, posto que também atingem o meio social.

\section{CONSIDERAÇÕES FINAIS}

Este trabalho objetivou conhecer como está organizada a rede formada pelos pedidos de remoção realizados pelos servidores técnicos-administrativos da UFAL. Nesse sentido, buscou-se descrever a rede de movimentação de servidores da instituição, comparar a estrutura e oportunidades de capacitação entre unidades/polos da universidade, verificar a distância social média entre os atores, apurar o potencial de crescimento da rede e identificar a atuação da PROGEP enquanto ator da rede.

Para construir a base teórica foram utilizados dois temas de grande relevância para a Administração Pública. O primeiro foi ARS que contempla indicadores a nível relacional, estrutural e de centralidade (FREEMAN, 1979; BRAND; VERSCHOORE, 2014), a partir dos 


\section{REDE DE MOVIMENTAÇÃO DOS SERVIDORES PÚBLICOS CIVIS DA UNIÃO: UM ESTUDO SOB A \\ PERSPECTIVA DA GESTÃO DE PESSOAS NA UFAL \\ DOI: http://dx.doi.org/10.5007/1983-4535.2019v12n2p160}

quais foram medidos os atributos dos atores e do fluxo da movimentação de pessoas sob a perspectiva de uma rede intraorganizacional. Já com relação ao segundo tema, Gestão de Pessoas, procedeu-se com a interpretação dos dados à luz de teorias sobre a gestão por competências (PIRES et al., 2005), as dificuldades para implementá-la no setor público (BERGUE, 2014) e a movimentação de pessoas como uma ferramenta a ser usada em favor da Administração Pública (DUTRA, 2013).

A metodologia adotada foi qualitativa e a coleta de dados foi feita por levantamento documental, entrevistas informais e semi-estruturadas junto a servidores da PROGEP a fim de obter informações acerca e da existência de normas internas e de procedimentos adotados para apreciação dos processos de remoção. Dados estatísticos sobre remoção foram solicitados por meio de processo administrativo, que resultou na entrega de planilha, que foi posteriormente processada pelo software UCINET.

Observando-se a composição da rede, Maceió foi identificado como o município que mais recebe pedidos de remoção por outros polos/unidades de ensino, enquanto Arapiraca aparece com o maior número de remoções protocoladas, mas ao mesmo tempo, é considerado o segundo maior centro da UFAL, pois recebe pedidos de remoção de todos os outros atores. Já as cidades do campus Sertão (Delmiro Gouveia e Santana do Ipanema) e o polo Penedo são os atores com os menores graus de entrada de toda a rede, ou seja, estes municípios praticamente não recebem processos de servidores interessados em ser removidos para lá, portanto, permutas nessas regiões se tornam mais escassas.

Quanto à distância social média entre os atores, o UCINET conseguiu apurar um diâmetro de 2 na rede. Nesse aspecto, seria ideal que todos os atores mantivessem uma conexão de grau 1 entre si, contudo, mesmo quando esse vínculo não existe, o grau nunca é superior a 2, pois a intermediação da PROGEP mantém a coesão na rede. Essa análise reflete também na importância da PROGEP enquanto ator da rede, pois segundo a teoria de Baran (1964), a exclusão de um vértice numa rede distribuída não compromete seu funcionamento, porém, sob a perspectiva de gestão de pessoas, verifica-se que retirar o setor responsável pelas decisões de RH acarreta prejuízo à instituição, que estabeleceu formalmente tais atribuições para este órgão por meio do seu regimento.

Concluiu-se, ainda, que existe potencial de crescimento da rede, isso porque atualmente a mesma opera com aproximadamente $66 \%$ de seu potencial, valor calculado a partir da densidade $(0,661)$. No caso em análise, reciprocidade dos vínculos é a principal 


\section{REDE DE MOVIMENTAÇÃO DOS SERVIDORES PÚBLICOS CIVIS DA UNIÃO: UM ESTUDO SOB A \\ PERSPECTIVA DA GESTÃO DE PESSOAS NA UFAL \\ DOI: http://dx.doi.org/10.5007/1983-4535.2019v12n2p160}

forma de expandir a rede e torna-la mais funcional, pois aqui, reciprocidade representa possibilidade de permuta, fator que potencializa remoções bem-sucedidas.

Em termos estruturais e em oportunidades de desenvolvimento profissional, Maceió figura em posição de destaque em relação aos demais municípios, pois concentra todos os cursos de doutoramento, quase todos os cursos de mestrado, e mais que o dobro dos cursos de graduação oferecidos nos demais polos/unidades de ensino. Esse é um dos fatores que contribuem para a aglomeração de pedidos para o campus A.C. Simões.

A partir dos resultados encontrados, sugere-se a implementação de um plano com cinco ações para promover o aprimoramento da rede. A primeira delas é a criação de novos cursos de graduação e pós-graduação para atender servidores do interior, evitando o deslocamento destes para qualificação. A segunda proposta é divulgar o banco de talentos da instituição para evitar remoções motivadas por exercício de atribuições fora das competências. A terceira é definir critérios objetivos para apreciar pedidos de remoção cujo contexto obriga a administração pública ao acolhimento destes. A quarta proposta é criar um banco de permuta que amplie a reciprocidade dos laços da rede. E por fim, a quinta e última ação é abertura periódica de edital de remoção devidamente respaldado em normas gerais e critérios preestabelecidos.

Do ponto de vista teórico, observou-se a utilização singular da interdisciplinaridade entre Análise de Redes e Gestão de Pessoas no contexto da Administração Pública, que se mostrou útil diante das situações de troca de recursos (humanos) entre atores de uma organização que encontra restrições normativas relacionadas a práticas de determinadas ações estratégicas, mas que, apesar das dificuldades, precisa implementá-las para obter o melhor desempenho possível na instituição.

A pesquisa apresenta limitações quanto ao universo e amostra, uma vez que não abarca as duas categorias de servidores pertencentes a UFAL, dessa forma, todas as análises e conclusões são válidas apenas para a realidade dos técnicos-administrativos, portanto, para futuras pesquisas, sugere-se um estudo sob a perspectiva dos docentes, que compõem aproximadamente a metade da população de servidores da instituição. Mais um ponto sobre o qual pode incidir estudo posterior é a replicação da metodologia deste trabalho em outras instituições para verificar as diferenças e similaridades a respeito dos procedimentos de remoção. E sob uma perspectiva interorganizacional, propõe-se transpor o foco da pesquisa 


\section{REDE DE MOVIMENTAÇÃO DOS SERVIDORES PÚBLICOS CIVIS DA UNIÃO: UM ESTUDO SOB A \\ PERSPECTIVA DA GESTÃO DE PESSOAS NA UFAL \\ DOI: http://dx.doi.org/10.5007/1983-4535.2019v12n2p160}

para os processos de redistribuição, a fim de observar os atributos da rede de movimentação de pessoas entre os diversos entes públicos a nível nacional.

Espera-se que o presente trabalho tenha contribuído com o desenvolvimento de políticas mais robustas na gestão de pessoas na UFAL, como também ensejar continuidade nas discussões acerca da complexidade do gerenciamento de recursos humanos no setor público.

\section{REFERÊNCIAS}

ABREU, Welles M.; GUIMARAES, Daniela R. Gestão do Orçamento Público. Brasília: ENAP, 2014. Disponível em < http://repositorio.enap.gov.br/handle/1/2207>. Acesso em 02 de ago. 2017.

ALEJANDRO, Velázquez A. O.; NORMAN, Aguilar G. Manual introdutório à análise de redes sociais: medidas de centralidade. Mexico: Universidad Autonoma Del Estado de México, 2005.

AMARAL, Helena K. Desenvolvimento de competências de servidores na administração pública brasileira. Revista do Serviço Público, v. 57, n. 4, 2006.

BARAN, Paul. Rand Memoranda on Distributed Communication, 1964. Disponível em: $<$ http://www.rand.org/publications/RM/baran.list.html>. Acesso em: 11 de mar. 2017.

BERGUE, Sandro T. Gestão estratégica de pessoas no setor público. São Paulo: Atlas, 2014.

BORDIN, Andrea S.; GONÇALVES, Alexandre L.; TODESCO, José L. Análise da colaboração científica departamental através de redes de coautoria. Perspectivas em Ciência da Informação, v. 19, n. 2, p. 37-52, 2014.

BRAND, Fabiane C.; VERSCHOORE, Jorge R. A utilização de medidas de Análise de Redes Sociais nas pesquisas em Administração. Revista Economia \& Gestão, v. 14, n. 35, p. 212237, 2014.

BRASIL. Decreto n. 5707, de 23 de fevereiro de 2006.

Lei n. 8112, de 11 de dezembro de 1990.

BRITTO JÚNIOR, Álvaro. F.; FERES JÚNIOR, Nazir. A utilização da técnica de entrevista em trabalhos científicos. Evidência, v. 7, n. 7, p. 237-250, 2011.

COLLIS, Jill; HUSSEY, Roger. Pesquisa em administração: um guia prático para alunos de graduação e pós-graduação. Porto Alegre: Bookman, 2005.

DUTRA, Joel S. Gestão de pessoas: modelos, processos, tendências e perspectivas. São Paulo: Atlas, 2013. 
FIALHO, Joaquim M. R. Análise de redes sociais: princípios, linguagem e estratégia de ação na gestão do conhecimento. Perspectivas em Gestão e Conhecimento, v. 4, número especial, p. 9-26, 2014.

FONSECA, João J. S. Metodologia da pesquisa científica. Fortaleza: UEC, 2002. Disponível em $<$ http://leg.ufpi.br/subsiteFiles/lapnex/arquivos/files/Apostila_-

_METODOLOGIA_DA_PESQUISA(1).pdf $>$. Acesso em: 07 ago. 2017.

FREEMAN, Linton C. Centrality in Social Networks: conceptual clarification. Social Networks, v. 1, p.215-239, 1979.

GERHARDT, Tatiana E.; SILVEIRA, Denise T. Métodos de pesquisa. Porto Alegre: Editora da UFRGS, 2009. Disponível em: < http://www.ufrgs.br/ cursopgdr/downloadsSerie/derad005 .pdf > . Acesso em: 09/08/2017.

HANNEMAN, Robert A.; RIDDLE, Mark. Introduction to social network methods, 2005. Disponível em: <https://www.researchgate.net/profile/Robert_Hanneman/publication/ 235737492_Introduction_to_Social_Network_Methods/links/0deec52261e1577e6c000000/Int roduction-to-Social-Network-Methods.pdf>. Acesso em: 12 de fev. 2017.

INGRAHAM, Patricia W.; RUBAII-BARRETT, Nadia. Human resource management as a core dimension of public administration. Foundations of Public Administration Series, $2007 . \quad$ Disponivel

em:

$<$ http://faculty.cbpp.uaa.alaska.edu/afgjp/PADM601\%20Fall\%202009/FPA-HRM-

Article.pdf $>$. Acesso em 26 ago. 2017.

MERRIAM, Sharan B. Qualitative research in practice: examples for discussion and analysis. São Francisco: Jossey-Bass, 2002.

OLIVEIRA, Maxwell F. Metodologia científica: um manual para a realização de pesquisas em Administração. Catalão: UFG, 2011.

OLIVEIRA, Pâmela M. M.; OLIVEIRA, Pamela T.; TAVARES, Bruno. Governança democrática em rede: o posicionamento das ONGs de Viçosa-MG. HOLOS, v. 1, n. 34, p. 103-117, 2018.

PANTOJA, Maria J.; CAMÕES, Marizaura R. S.; BERGUE, Sandro T. Gestão de pessoas: bases teóricas e experiências no setor público. Brasília: ENAP, 2010. Disponível em: $<$ http://repositorio.enap.gov.br/handle/1/514>. Acesso em: 6 ago. 2017.

PIRES, Alexandre $K$. et al. Gestão por competências em organizações de governo. Brasília: Enap, 2005. 100 p. Disponível em: <http://repositorio.enap.gov.br/handle/1/383>. Acesso em: 04 ago. 2017.

SCOTT, John. Social network analysis. Sage, 2012.

UFAL. Plano de desenvolvimento institucional 2013-2017. Disponível em: $<$ www.ufal.edu.br/transparencia/institucional/plano-de-desenvolvimento $>$. Acesso em: 06 de março de 2017. 